\title{
Fabrication of GaN with Buried Tungsten (W) Structures Using Epitaxial Lateral Overgrowth (ELO) via LP-MOVPE
}

Hideto Miyake ${ }^{1}$, Motoo Yamaguchi', Masahiro Haino', Atsushi Motogaito ${ }^{1}$, Kazumasa Hiramatsu ${ }^{1}$, Shingo Nambu ${ }^{2}$, Yasutoshi Kawaguchi ${ }^{2}$, Nobuhiko Sawaki ${ }^{2}$, Yasushi Iyechika ${ }^{3}$, Takayoshi Maeda ${ }^{3}$ and Isamu Akasaki ${ }^{4}$

${ }^{1}$ Dept. of Electrical and Electronic Engineering, Mie University, 1515 Kamihama, Tsu, Mie, 514-8507, Japan

${ }^{2}$ Dept. of Electronic Engineering, Nagoya University, Furo, Chikusa, Nagoya, 464-8603

${ }^{3}$ Tsukuba Research Laboratory, Sumitomo Chemical Co., Ltd, 6 Kitahara, Tsukuba, 3003294, Japan

${ }^{4}$ Dept. of Electrical and Electronic Engineering, Meijo University, 1-501 Shiogamaguchi, Tempaku, Nogoya 468-8502, Japan

\section{ABSTRACT}

A buried tungsten (W) mask structure with GaN is successfully obtained by epitaxial lateral overgrowth (ELO) technique via low-pressure metalorganic vapor phase epitaxy (LP-MOVPE). The selectivity of GaN growth on the window region vs. the mask region is good. An underlying GaN with a striped $\mathrm{W}$ metal mask is easily decomposed above $500{ }^{\circ} \mathrm{C}$ by the $\mathrm{W}$ catalytic effect, by which radical hydrogen is reacted with $\mathrm{GaN}$. It is difficult to bury the W mask because severe damage occurs in the GaN epilayer under the mask. It is found that an underlying AlGaN/GaN layer with a narrow $\mathrm{W}$ stripe mask width (mask/window $=2 / 2 \mu \mathrm{m}$ ) leads the ELO GaN layer to be free from damage, resulting in an excellent $\mathrm{W}$-buried structure.

\section{INTRODUCTION}

The group III-nitride semiconductors are promising materials for applications in opto-electronic devices such as light emitting diodes and laser diodes [1], and in field effect transistors[2]. Although these devices are fabricated mainly on sapphire substrate, it is not easy to achieve a high performance because of a high dislocation density in the crystal originating from the large differences in lattice constants between $\mathrm{GaN}$ and sapphire. An attempt to reduce the dislocation density of crystal using epitaxial lateral overgrowth (ELO) technique was employed in GaAs[3]. Recently, Usui et al. achieved a thick GaN epitaxial layer with a dislocation density as low as $10^{7} \mathrm{~cm}^{-2}$ using ELO by hydride vapor phase epitaxy (HVPE) [4].

$\mathrm{SiO}_{2}$ or $\mathrm{SiN}_{\mathrm{x}}$ insulator has been normally used as the mask material for ELO-GaN. Besides those materials, ELO-GaN with tungsten (W) metal mask was investigated to fabricate optical and electronic devices with higher performance. It was reported that the ELO-GaN layers are successfully obtained with the $\mathrm{W}$ mask as well as $\mathrm{SiO}_{2}$ or $\mathrm{SiN}_{\mathrm{x}}$ masks via HVPE and have a better crystalline quality than that with $\mathrm{SiO}_{2}$ mask[5]. 
In the ELO of GaAs, the W metal mask was employed and the W-buried structure was obtained to fabricate a permeable base transistor (PBT) or static induction transistor (SIT) [6]. The fabrication of the electronic devices such as PBTs or SITs via the ELO technique in $\mathrm{GaN}$ is very attractive for the use as high power and/or high frequency devices under crucial environments such as at high temperature or at highly radioactive environment. Recently, Kawaguchi et al. performed the selective area growth (SAG) of $\mathrm{GaN}$ using the $\mathrm{W}$ mask by metalorganic vapor phase epitaxy (MOVPE) and achieved high selectivity[7].

In this work, we demonstrate the ELO of GaN using the W mask by MOVPE and obtain the W-buried structure with GaN. The characteristics of ELO-GaN were also investigated by means of atomic force microscopy (AFM) and x-ray rocking curve (XRC).

\section{EXPERIMENTAL PROCEDURE}

MOVPE of GaN using a W mask was performed on two types of samples. The sample is a $4.0 \mu$ m-thick (0001) GaN epilayer, which had been grown on sapphire (0001) substrate using an GaN low temperature buffer layer by MOVPE. Another sample is a 40-100 nm thick AlGaN epilayer on the $4.0 \mu \mathrm{m}$-thick (0001) GaN epilayer. The $30-50$ $\mathrm{nm}$ thick $\mathrm{W}$ mask was deposited by electron beam evaporation method. Stripe windows of 2 or $5 \mu \mathrm{m}$ width with a periodicity of 4 or $10 \mu \mathrm{m}$, respectively, were developed in the <1100> direction of the GaN by conventional photolithographic method and then wet etching with a hydrogen peroxide $\left(\mathrm{H}_{2} \mathrm{O}_{2}\right) \square$ and an ammonia solution. The source gases were trimethylgallium (TMG) and ammonia $\left(\mathrm{NH}_{3}\right)$, respectively. Hydrogen gas was used as a carrier gas during the growth process. ELO of GaN was performed by LP (low pressure) - MOVPE system with a horizontal reactor. Two steps ELO technique was carried out to bury $\mathrm{W}$ mask completely. In the first step, a low temperature $\left(950^{\circ} \mathrm{C}\right)$ growth was performed for $30 \mathrm{~min}$ to prevent the underlying $\mathrm{GaN}$ from being decomposed. In the second step, a high temperature $\left(1050^{\circ} \mathrm{C}\right)$ growth was done for 90 min to bury $\mathrm{W}$ mask. Here, it is expected in both steps that the GaN with $\{1122\}$ facets are grown selectively in the first step and that the $\mathrm{W}$ mask is easily covered with GaN because of the fast ELO rate at a higher growth temperature in the second step, based on the results of ELO-GaN using $\mathrm{SiO}_{2}$ mask[8]. 


\section{RESULTS AND DISCUSSION}

In order to investigate effects of the $\mathrm{W}$ mask which affects the underlying $\mathrm{GaN}$ and $\mathrm{AlGaN}$ layers in hydrogen ambient at a high temperature, we attempted thermal annealing at 500 to $700{ }^{\circ} \mathrm{C}$ of $\mathrm{GaN}$ and $\mathrm{AlGaN}$ with a striped W mask. Consequently, the surface of $\mathrm{GaN}$ window regions was roughened, including $\mathrm{Ga}$ droplets and $\mathrm{GaN}$ whiskers. This phenomenon occurred above $500^{\circ} \mathrm{C}$. It is reported that an underlying $\mathrm{GaN}$ layer with a striped $\mathrm{SiO}_{2}$ mask is decomposed higher than $900{ }^{\circ} \mathrm{C}$ in hydrogen ambient and there is no damage less than $900{ }^{\circ} \mathrm{C}[9]$. Therefore, the $\mathrm{W}$ mask works as a catalyst to enhance decomposition of $\mathrm{GaN}$. It is thought that $\mathrm{W}$ may produce radical hydrogen that can decompose $\mathrm{GaN}$ easily. On the other hand, the surface of $\mathrm{AlGaN}$ is not roughened even though the annealing temperature is $700^{\circ} \mathrm{C}$. Any Ga droplets and $\mathrm{GaN}$ whiskers are not observed on the $\mathrm{AlGaN}$ layer. Hence, the catalytic effect of the $\mathrm{W}$ mask does not work in the AlGaN layer. Thus, it is found that the top AlGaN layer plays an important role to protect the bottom $\mathrm{GaN}$ from decomposition.

The ELO was performed using the $\mathrm{W}$ mask with stripe windows of $5 \mu \mathrm{m}$ width. Figure 1 shows the cross sectional SEM images of ELO GaN with the underlying GaN (a) and $\mathrm{AlGaN} / \mathrm{GaN}$ (b). About $5 \mu \mathrm{m}$-thick ELO GaN layers are obtained in both cases.

(a)
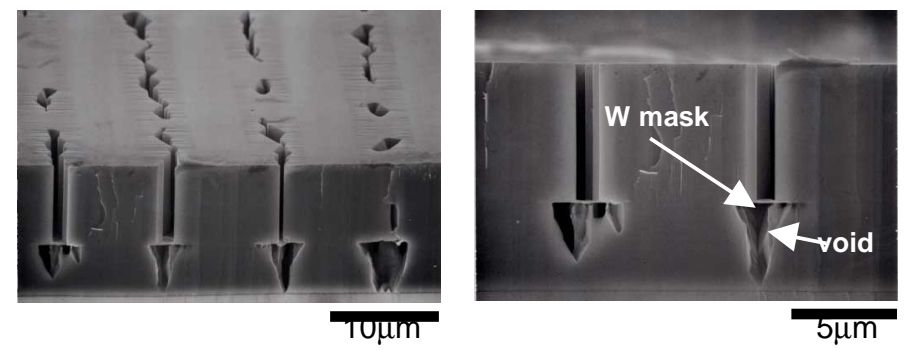

(b)
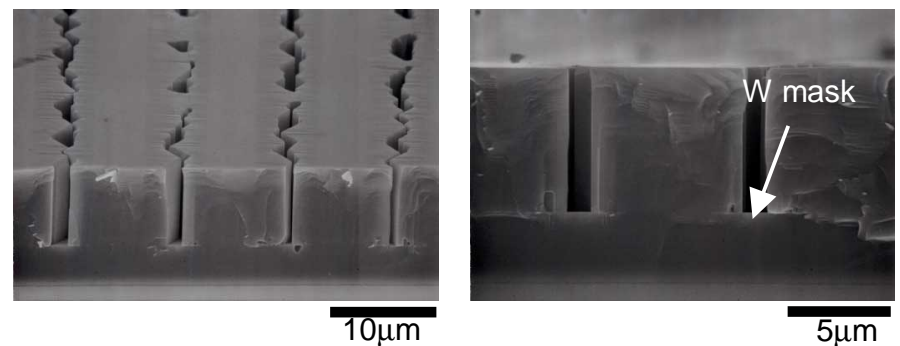

Figure 1 The cross sectional SEM photographs of ELO GaN with underlying $G a N(a)$ and $A l G a N(b)$. The width of stripe window is $5 \mu \mathrm{m}$. 
Large voids occurs under the $\mathrm{W}$ mask as seen in Fig.1(a), indicating that the underlying $\mathrm{GaN}$ is decomposed during the ELO process owing the catalytic effect of the W mask. On the contrary, there are no voids in Fig.1(b). Therefore, the underlying AlGaN layer can prevent the bottom GaN from being decomposed.

The $\mathrm{W}$ masks are not buried completely after the growth process in the case of the $5 \mu \mathrm{m}$ wide mask. The width of stripe was narrowed to bury the $\mathrm{W}$ mask completely. Figure 2 shows the cross sectional SEM images of ELO GaN with the underlying $\mathrm{AlGaN} / \mathrm{GaN}$ layer. The widths of mask and window are $2 \mu \mathrm{m}$ and $2 \mu \mathrm{m}$, respectively. This result clearly shows that the W mask is buried completely and the surface is smooth.

To characterize the surface morphology and the tilting of c-axis of the ELO GaN, we carried out x-ray diffraction and atomic force microscope (AFM). Figure 3 shows the results of AFM for the sample of Fig. 2. In this figure, atomic step structures with 0.1 $\mu \mathrm{m}$-width and $0.3 \mathrm{~nm}$-height are observed. This means that the step flow growth occurs in the ELO of the GaN layer. Figure 4 shows the XRCs of the (0004) reflection for $\phi=0$ ${ }^{\circ}$ and $90^{\circ}$ of the ELO GaN layer with the stripe directions of the masks along the direction of $\langle 1100\rangle$, where $\phi$ is the azimuth angle between the stripe direction of the mask and the scattering plane. The full width of half maximum (FWHM) is about 230 $\operatorname{arcsec}$ for each $\phi$. There are no satellite peaks near the main peak, which suggests no tilting of c-axis. Thus, it is found that the ELO GaN with the $\mathrm{W}$ mask has high crystalline quality as well as a good surface morphology.

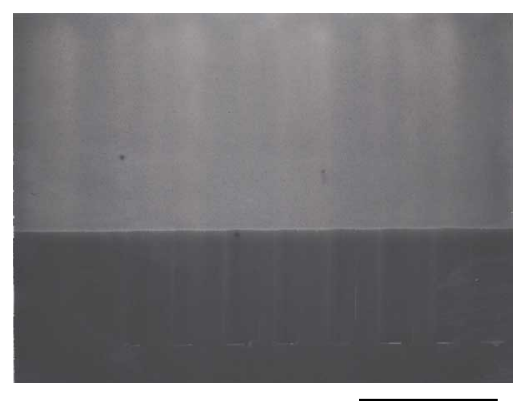

$10 \mu \mathrm{m}$

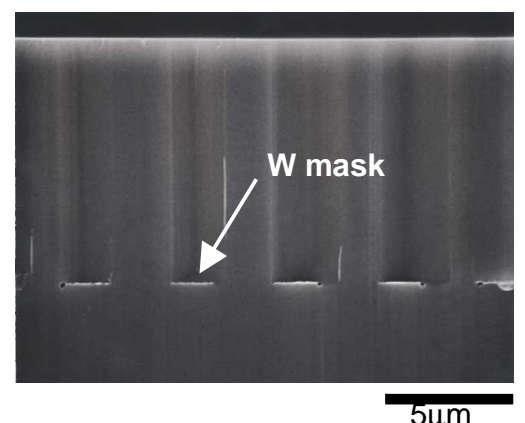

$5 \mu \mathrm{m}$

Figure 2 The cross sectional SEM photographs of ELO GaN with underlying AlGaN. The width of stripe window is $2 \mu \mathrm{m}$. 


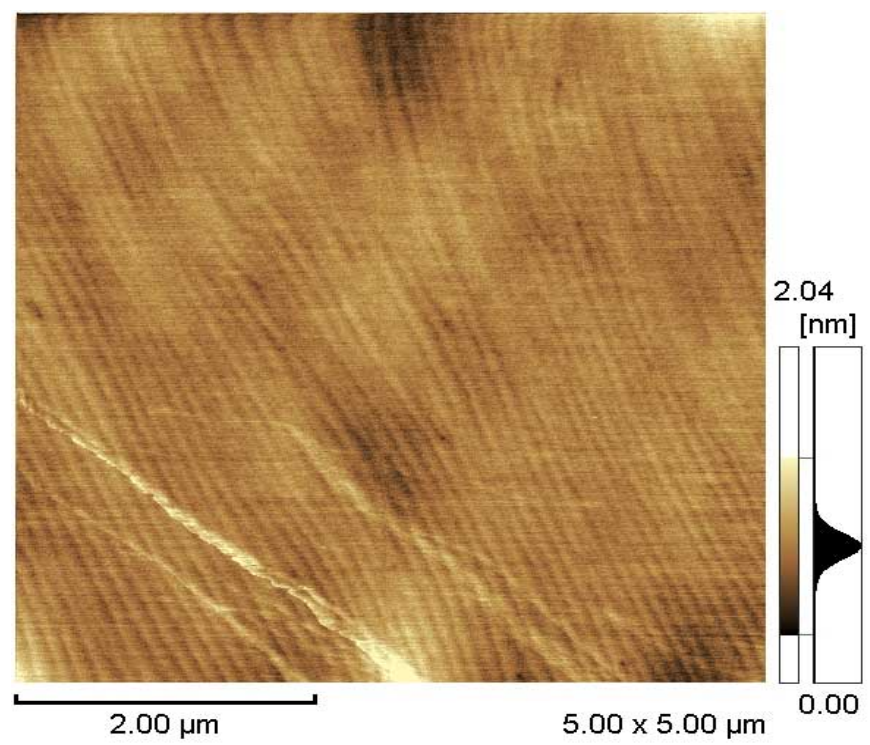

Figure 3 The results of AFM for the sample of Fig.2.

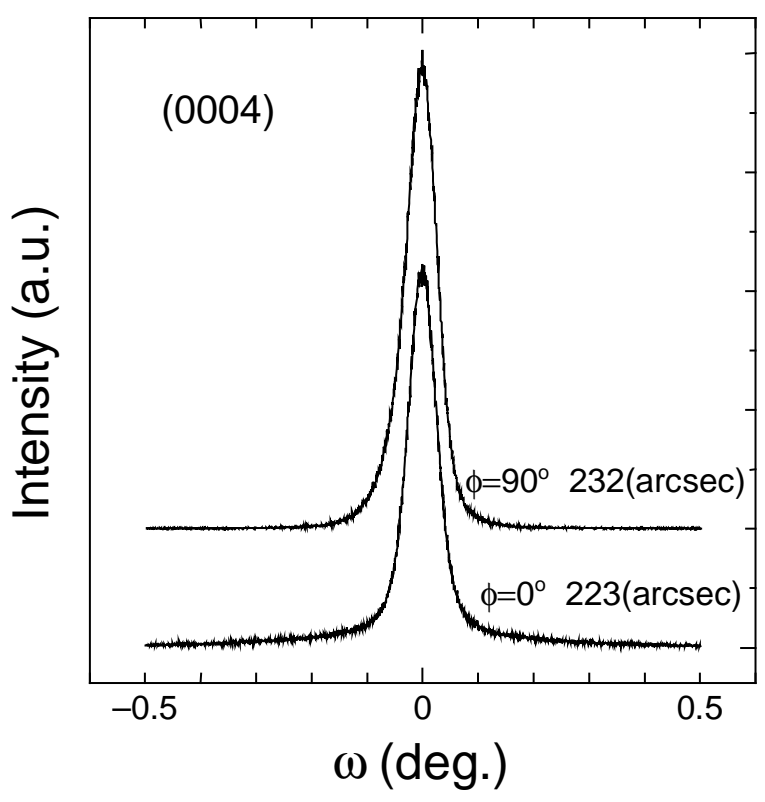

Figure 4 The XRCs of the (0004) reflection of the sample of Fig.2. 


\section{CONCLUSION}

The two step ELO of GaN using W mask was performed by LP- MOVPE. GaN with a striped $\mathrm{W}$ metal pattern is easily decomposed above $500{ }^{\circ} \mathrm{C}$ by the $\mathrm{W}$ catalytic effect that causes radical hydrogen to attack the GaN layer. It is difficult to bury the W mask because severe damage occurs in the GaN epilayer under the mask. By employing the $\mathrm{AlGaN} / \mathrm{GaN}$ underlying layer with the narrow $\mathrm{W}$ stripe mask of $2 \mu \mathrm{m}$, the excellent $\mathrm{W}$-buried structure can be obtained free from damage.

\section{ACKNOWLEDGEMENTS}

This work was partly supported by "Research for the Future" program of Atomic Scale and Interface Dynamics of JSPS, "Light of the 21st Century" program of NEDO, the Proposed-Based R\&D Program of NEDO (97S02-015) and Grant-in-Aid for Scientific Research from the Ministry of Education, Science, Sports and Culture (No.11450012).

\section{REFERENCES}

1 S. Nakamura, M. Senoh, S. Nagahama, N. Iwasa, T. Yamada, T. Matsushita, H. Kiyoku, Y. Sugimoto, T. Kozaki, H. Umemoto, M. Sano and K. Chocho, Jpn. J. Appl. Lett. 36 (1997) L1568.

2 R. Vetury, H. Marchand, G. Parish, P. T. Fini, J. P. Ibbetson, S. Keller, J. S. Speck, S. P. DenBaars and U. K. Mishra, Inst. Phys. Conf. Ser. No. 162: Chapter5 (1999) 177.

3 Y. Ujiie and T. Nishinaga, Jpn. J. Appl. Phys. 28 (1989) L327.

4 A. Usui, H. Sunakawa, A. Sakai and A. Yamaguchi, Jpn. J. Appl. Phys. 36 (1997) L899.

5 H. Sone, S. Nambu, Y. Kawaguchi, M. Yamaguchi, H. Miyake, K. Hiramatsu, Y. Iyechika, T. Maeda and N. Sawaki, Jpn. J. Appl. Phys. 38 (1999) L356.

6 H. Asai, S. Adachi, S. Ando and K. Oe, J. Appl. Phys. 55 (1984) 3868

7 Y. Kawaguchi, S. Nambu, H. Sone, M. Yamaguchi, H. Miyake, K. Hiramatsu, and N. Sawaki, Jpn. J. Appl. Phys. 37 (1998) L845.

8 H. Miyake, A. Motogaito and K. Hiramatsu, Jpn. J. Appl. Phys. 38 (1999) L1000.

9 K. Hiramatsu, H. Matsushima, H. Hanai and N. Sawaki : Mat. Res. Soc. Symp. Proc. 482 (1998) 991. 